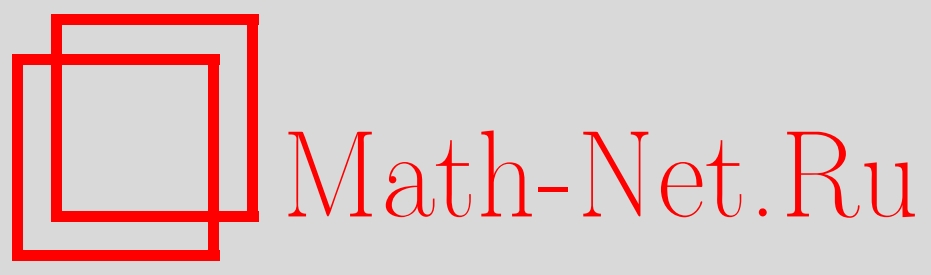

М. Х. Сабуров, О сюръективности квадратичных стохастических операторов на симплексе, Матем. заметки, 2016, том 99, выпуск 4, 635-639

DOI: https://doi.org/10.4213/mzm11063

Использование Общероссийского математического портала Math-Net.Ru подразумевает, что вы прочитали и согласны с пользовательским соглашением http://www . mathnet.ru/rus/agreement

Параметры загрузки:

IP : 54.81 .137 .203

26 апреля 2023 г., 04:16:52

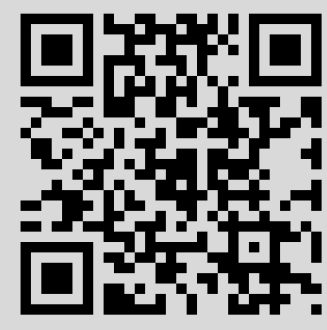




\section{О сюръективности}

\section{квадратичных стохастических операторов на симплексе}

\section{М. Сабуров}

1. Введение. Пусть $\left\{\mathbf{e}_{k}\right\}_{k=1}^{m}-$ стандартный базис в $\mathbb{R}^{m}$ и

$$
\mathbb{S}^{m-1}=\left\{\mathbf{x}=\left(x_{1}, \ldots, x_{m}\right) \in \mathbb{R}^{m}: \sum_{k=1}^{m} x_{k}=1, x_{k} \geqslant 0 \text { для всех } k=1, \ldots, m\right\}
$$

- стандартный $(m-1)$-мерный симплекс. Элемент такого симплекса называется стохастическим вектором. Напомним, что квадратная матрица $\mathbb{P}=\left(p_{i j}\right)_{i, j=1}^{m}$ называется стохастической, если каждая ее строка является стохастическим вектором. Пусть $\mathscr{L}: \mathbb{S}^{m-1} \rightarrow$ $\mathbb{S}^{m-1}$ - линейный стохастический оператор (марковский оператор) с квадратной стохастической матрицей $\mathbb{P}$, т.е. $\mathscr{L}(\mathbf{x})=\mathbf{x} \mathbb{P}$. Легко видеть, что линейный стохастический оператор $\mathscr{L}: \mathbb{S}^{m-1} \rightarrow \mathbb{S}^{m-1}$ сюръективен, если и только если он биективен. Действительно, непосредственные вычисления показывают, что если оператор $\mathscr{L}: \mathbb{S}^{m-1} \rightarrow \mathbb{S}^{m-1}$ сюръективен, то для каждого $i$ найдется такое $j$, что $\mathscr{L}^{-1}\left(\mathbf{e}_{i}\right)=\mathbf{e}_{j}$, где $\mathscr{L}^{-1}\left(\mathbf{e}_{i}\right)-$ прообраз вершины $\mathbf{e}_{i}$ симплекса $\mathbb{S}^{m-1}$. Следовательно, сюръективные линейные стохастические операторы симплекса - это в точности операторы перестановки. Аналогичная задача для квадратичных стохастических операторов (нелинейных марковских операторов) на симплексе открыта [1]. В общем случае сюръективность квадратичных операторов тесно связана с нелинейными проблемами оптимизации [2], и описание таких операторов - трудная задача. В классе всех квадратичных операторов можно выделить класс операторов специального вида, а именно, так называемых квадратичных стохастических операторов, которым соответствуют кубические стохастические матрицы; такие операторы имеют удивительные приложения в генетике популяций (см. [3]-[5]), а также в теории многоагентных систем (см. [6], [7]). В этой статье получен критерий сюръективности квадратичных стохастических операторов с кубическими стохастическими матрицами; тем самым решена задача 2.36 , поставленная в [1].

2. Сюръективные квадратичные стохастические операторы. Пусть $\mathbf{I}_{m}=\{1, \ldots$, $m\}$ - конечное множество. Мы обозначаем дополнение любого подмножества $\alpha \subset \mathbf{I}_{m}$ через $\bar{\alpha}: \bar{\alpha}=\mathbf{I}_{m} \backslash \alpha$. Для стохастического вектора $\mathbf{x} \in \mathbb{S}^{m-1}$ мы полагаем

$$
\operatorname{supp}(\mathbf{x})=\left\{i \in \mathbf{I}_{m}: x_{i}>0\right\} \quad \text { и } \quad \operatorname{null}(\mathbf{x})=\left\{i \in \mathbf{I}_{m}: x_{i}=0\right\} .
$$

Ясно, что $\operatorname{supp}(\mathbf{x}) \cup \operatorname{null}(\mathbf{x})=\mathbf{I}_{m}$ и $\operatorname{supp}(\mathbf{x}) \cap \operatorname{null}(\mathbf{x})=\varnothing$ для всякого $\mathbf{x} \in \mathbb{S}^{m-1}$. Гранъю симплекса $\mathbb{S}^{m-1}$ называется любое множество вида $\Gamma_{\alpha}=\operatorname{conv}\left\{e_{i}\right\}_{i \in \alpha}$, где $\alpha \subset \mathbf{I}_{m}$, a conv $(A)$ обозначает выпуклую оболочку множества $A$. Внутренность грани $\Gamma_{\alpha}$ определяется как

$$
\operatorname{int} \Gamma_{\alpha}=\left\{\mathbf{x} \in \Gamma_{\alpha}: x_{i}>0 \text { для всех } i \in \alpha\right\} .
$$

Пусть $\mathscr{P}=\left(p_{i j k}\right)_{i, j, k=1}^{m}-$ кубическая матрица. Кубическая матрица $\mathscr{P}$ называется $\mathrm{cmo-}$ хастической, если каждый вектор $\mathbf{p}_{i j} \bullet=\left(p_{i j 1}, \ldots, p_{i j m}\right)$ является стохастическим для всех $i, j=1, \ldots, m$. С кубической стохастической матрицей $\mathscr{P}=\left(p_{i j k}\right)_{i, j, k=1}^{m}$ связан квадратичный стохастический оператор $\mathscr{Q}: \mathbb{S}^{m-1} \rightarrow \mathbb{S}^{m-1}$, определенный правилом

$$
\mathscr{Q}(\mathbf{x})=\sum_{i, j=1}^{m} x_{i} x_{j} \mathbf{p}_{i j} \bullet
$$

Работа выполнена при финансовой поддержке Министерства образования Малайзии (грант FRGS14-141-0382)

DOI: $10.4213 / \mathrm{mzm} 11063$

(C) M. Сабуров, 2016 
для любого $\mathbf{x} \in \mathbb{S}^{m-1}$. Без потери общности можно считать, что $\mathbf{p}_{i j} \bullet=\mathbf{p}_{j i} \bullet$ для всех $i, j=$ $1, \ldots, m$. Предположим, что $\mathbf{x} \in \mathbb{S}^{m-1}$ и $\operatorname{supp}(\mathbf{x})=\alpha$. Тогда для всякого $\mathbf{x} \in \operatorname{int} \Gamma_{\alpha}$ имеем

$$
\mathscr{Q}(\mathbf{x})=\sum_{i, j \in \alpha} x_{i} x_{j} \mathbf{p}_{i j \bullet}
$$

Отсюда мы немедленно получаем следующий результат.

ПредЛОЖЕнИЕ 1. Справедливы следующие утверждения:

(i) $\operatorname{supp}(\mathscr{Q}(\mathbf{x}))=\bigcup_{i, j \in \operatorname{supp}(\mathbf{x})} \operatorname{supp}\left(\mathbf{p}_{i j} \bullet\right.$;

(ii) $\operatorname{null}(\mathscr{Q}(\mathbf{x}))=\bigcap_{i, j \in \operatorname{supp}(\mathbf{x})} \operatorname{null}\left(\mathbf{p}_{i j} \bullet\right.$;

(iii) $\mathscr{Q}\left(\operatorname{int} \Gamma_{\alpha}\right) \subset \operatorname{int} \Gamma_{\beta}$, где $\beta=\bigcup_{i, j \in \alpha} \operatorname{supp}\left(\mathbf{p}_{i j} \bullet\right.$;

(iv) $\mathscr{Q}\left(\operatorname{int} \Gamma_{\alpha}\right) \subset \operatorname{int} \Gamma_{\beta}$ тогда и только тогда, когда $\mathscr{Q}\left(\mathbf{x}^{(0)}\right) \in \operatorname{int} \Gamma_{\beta}$ для некоторого $\mathbf{x}^{(0)} \in$ $\operatorname{int} \Gamma_{\alpha}$.

В теории классических марковских цепей важную роль играют поглощающие состояния. По аналогии мы определяем понятие поглощающих множеств для квадратичных стохастических операторов.

ОПРеДЕлЕНиЕ 1. Множество $\alpha \subset \mathbf{I}_{m}$ называется поглощающим, если

$$
\bar{\alpha}=\bigcap_{i, j \in \alpha} \operatorname{null}\left(\mathbf{p}_{i j \bullet}\right) .
$$

Для каждого поглощающего множества $\alpha \subset \mathbf{I}_{m}$ кубическая матрица $\mathscr{P}_{\alpha}=\left(p_{i j k}\right)_{i, j, k \in \alpha}$ также является стохастической, и с ней можно связать квадратичный стохастический оператор $\mathscr{Q}_{\alpha}: \Gamma_{\alpha} \rightarrow \Gamma_{\alpha}$, определенный правилом

$$
\mathscr{Q}_{\alpha}(\mathbf{x})=\sum_{i, j \in \alpha} x_{i} x_{j} \mathbf{p}_{i j} \bullet \quad \text { для } \quad \mathbf{x} \in \Gamma_{\alpha} .
$$

Ясно, что $\left.\mathscr{Q}\right|_{\Gamma_{\alpha}}=\mathscr{Q}_{\alpha}$.

ПреДЛОЖенИЕ 2. Для любого множества $\alpha \subset \mathbf{I}_{m}$ следующие условия равносильны:

(i) множество а является поглощающим;

(ii) $\mathscr{Q}\left(\operatorname{int} \Gamma_{\alpha}\right) \subset \operatorname{int} \Gamma_{\alpha}$;

(iii) $\mathscr{Q}\left(\mathbf{x}^{(0)}\right) \in \operatorname{int} \Gamma_{\alpha}$ для некоторого $\mathbf{x}^{(0)} \in \operatorname{int} \Gamma_{\alpha}$.

Доказательство. Мы проведем доказательство по схеме (i) $\Rightarrow$ (ii) $\Rightarrow$ (iii) $\Rightarrow$ (i).

(i) $\Rightarrow$ (ii). Предположим, что множество $\alpha$ поглощающее. Тогда

$$
\alpha=\mathbf{I}_{m} \backslash \bar{\alpha}=\mathbf{I}_{m} \backslash \bigcap_{i, j \in \alpha} \operatorname{null}\left(\mathbf{p}_{i j} \bullet\right)=\bigcup_{i, j \in \alpha} \operatorname{supp}\left(\mathbf{p}_{i j} \bullet\right)
$$

По предложению 1 имеем $\mathscr{Q}\left(\operatorname{int} \Gamma_{\alpha}\right) \subset \operatorname{int} \Gamma_{\alpha}$.

Импликация (ii) $\Rightarrow$ (iii) очевидна.

(iii) $\Rightarrow$ (i). Пусть

$$
\mathscr{Q}\left(\mathbf{x}^{(0)}\right)=\sum_{i, j \in \alpha} x_{i}^{(0)} x_{j}^{(0)} \mathbf{p}_{i j} \bullet \in \operatorname{int} \Gamma_{\alpha} .
$$

Тогда $\bigcup_{i, j \in \alpha} \operatorname{supp} \mathbf{p}_{i j \bullet}=\alpha$. Следовательно, $\bar{\alpha}=\bigcap_{i, j \in \alpha} \operatorname{null}\left(\mathbf{p}_{i j} \bullet\right.$.

Предложение доказано.

ПредлОЖЕниЕ 3. Если все не более чем двухэлементные подмножества а множества $\mathbf{I}_{m}$ являются поглощающими, то любое подмножество множества $\mathbf{I}_{m}$ является поглощающим. 
ДоКАЗАТЕльство. Предположим, что любое множество $\alpha$ с $|\alpha| \leqslant 2$ является поглощающим. Тогда $\mathbf{p}_{i i \bullet}=\mathbf{e}_{i}$ и $\mathbf{p}_{i j} \bullet \in \operatorname{conv}\left\{\mathbf{e}_{i}, \mathbf{e}_{j}\right\}$ для всех $i, j \in \mathbf{I}_{m}$. Из включения null $\left(\mathbf{p}_{i j} \bullet\right) \supset$ $\mathbf{I}_{m} \backslash\{i, j\}$ вытекает, что

$$
\bigcap_{i, j \in \gamma} \operatorname{null}\left(\mathbf{p}_{i j \bullet}\right)=\bigcap_{i \in \gamma} \operatorname{null}\left(\mathbf{p}_{i i \bullet}\right) \cap \bigcap_{i \neq j} \operatorname{null}\left(\mathbf{p}_{i j} \bullet\right)=\mathbf{I}_{m} \backslash \gamma=\bar{\gamma}
$$

для любого $\gamma \subset \mathbf{I}_{m}$. Это означает, что $\gamma$ является поглощающим, что и требовалось.

Лемма 1. Если любое не более чем двухэлементное множество $\alpha \subset \mathbf{I}_{m}$ является поглощающим, то квадратичный стохастический оператор $\mathscr{Q}: \mathbb{S}^{m-1} \rightarrow \mathbb{S}^{m-1}$ сюрвективен.

ДокАЗАТЕЛЬСтво. В силу предложений 2 и 3 квадратичный стохастический оператор $\mathscr{Q}: \mathbb{S}^{m-1} \rightarrow \mathbb{S}^{m-1}$ отображает каждую грань симплекса в себя. Нам нужно показать, что этот оператор сюръективен. Применим математическую индукцию по размерности симплекса. В случае $m=2$ сюръективность оператора очевидна. Предположим, что сюръективность доказана для $m \leqslant n-1$. Докажем ее для $m=n$. По предположению индукции оператор $\mathscr{Q}: \partial \mathbb{S}^{n-1} \rightarrow \partial \mathbb{S}^{n-1}$ сюръективен. Покажем, что для любого $\mathbf{y} \in \operatorname{int} \mathbb{S}^{n-1}$ множество $\mathscr{Q}^{-1}(\mathbf{y})$ непусто. Предположим, что это не так и $\mathscr{Q}^{-1}(\mathbf{y})$ является пустым множеством. Рассмотрим отображение $g: \mathbb{S}^{n-1} \backslash\{\mathbf{y}\} \rightarrow \partial \mathbb{S}^{n-1}$, которое переводит каждую точку $\mathbf{z} \in \mathbb{S}^{n-1} \backslash\{\mathbf{y}\}$ в точку пересечения луча, идущего из $\mathbf{z}$ в направлении $\mathbf{y}$, с границей симплекса. Легко проверить, что отображение $\mathscr{F}: \mathbb{S}^{n-1} \rightarrow \mathbb{S}^{n-1}, \mathscr{F}=g \circ \mathscr{Q}$, не имеет неподвижных точек. Это противоречит теореме Брауэра о неподвижных точках ${ }^{1}$, что и доказывает лемму.

Теорема 1. Квадратичный стохастический оператор $\mathscr{Q}: \mathbb{S}^{m-1} \rightarrow \mathbb{S}^{m-1}$ сюрвективен тогда и только тогда, когда для некоторой перестановки $\pi$ множества $\mathbf{I}_{m}$ выполнены следующие условия:

(i) $\mathscr{Q}^{-1}\left(\mathbf{e}_{i}\right)=\mathbf{e}_{\pi(i)}$ для каждого $i \in \mathbf{I}_{m}$;

(ii) $\mathscr{Q}^{-1}\left(\operatorname{int}\left(\Gamma_{\mathbf{e}_{i} \mathbf{e}_{j}}\right)\right)=\operatorname{int}\left(\Gamma_{\mathbf{e}_{\pi(i)}} \mathbf{e}_{\pi(j)}\right) \partial л я л ю б ы x i, j \in \mathbf{I}_{m}\left(\right.$ здесъ $\left.\Gamma_{\mathbf{e}_{i} \mathbf{e}_{j}}=\operatorname{conv}\left\{\mathbf{e}_{i}, \mathbf{e}_{j}\right\}\right)$.

ДокАзАтельство. Достаточность вытекает из леммы 1.

Докажем необходимость. Предположим, что оператор $\mathscr{Q}$ сюръективен. Пусть $\mathscr{Q}^{-1}\left(\mathbf{e}_{i}\right)-$ прообраз вектора $\mathbf{e}_{i}$. Положим

$$
\operatorname{supp}\left(\mathscr{Q}^{-1}\left(\mathbf{e}_{i}\right)\right)=\bigcup_{\mathbf{x} \in \mathscr{Q}^{-1}\left(\mathbf{e}_{i}\right)} \operatorname{supp}(\mathbf{x}), \quad \Gamma_{\operatorname{supp}\left(\mathscr{Q}^{-1}\left(\mathbf{e}_{i}\right)\right)}=\operatorname{conv}\left\{e_{j}\right\}_{j \in \operatorname{supp}\left(\mathscr{Q}^{-1}\left(\mathbf{e}_{i}\right)\right)} .
$$

Из предложения 1 вытекает, что $\mathscr{Q}\left(\Gamma_{\operatorname{supp}\left(\mathscr{Q}^{-1}\left(\mathbf{e}_{i}\right)\right)}\right)=\mathbf{e}_{i}$. Следовательно,

$$
\left\{\mathbf{e}_{j}\right\}_{j \in \operatorname{supp}\left(\mathscr{Q}^{-1}\left(\mathbf{e}_{i}\right)\right)} \subset \mathscr{Q}^{-1}\left(\mathbf{e}_{i}\right) \quad \text { для любого } i \in \mathbf{I}_{m} .
$$

Значит, $\left|\operatorname{supp}\left(\mathscr{Q}^{-1}\left(\mathbf{e}_{i}\right)\right)\right|=1$. Это означает, что для некоторой перестановки $\pi$ множества $\mathbf{I}_{m}$ имеем $\mathscr{Q}^{-1}\left(\mathbf{e}_{i}\right)=\mathbf{e}_{\pi(i)}$ при любом $i \in \mathbf{I}_{m}$. Пусть $\mathbf{y} \in \operatorname{int} \Gamma_{\mathbf{e}_{i} \mathbf{e}_{j}}$ и $\mathbf{x} \in \mathscr{Q}^{-1}(\mathbf{y})$. Согласно предложению 1 имеет место включение

$$
\mathscr{Q}\left(\operatorname{int}\left(\Gamma_{\operatorname{supp}(\mathbf{x})}\right)\right) \subset \operatorname{int} \Gamma_{\mathbf{e}_{i} \mathbf{e}_{j}} .
$$

Если $k \in \operatorname{supp}(\mathbf{x}) \backslash\{\pi(i), \pi(j)\} \neq \varnothing$, то $\mathscr{Q}\left(\mathbf{e}_{k}\right) \in \overline{\mathscr{Q}\left(\operatorname{int}\left(\Gamma_{\operatorname{supp}(\mathbf{x})}\right)\right)} \subset \Gamma_{\mathbf{e}_{i} \mathbf{e}_{j}}-$ противоречие. Значит, $\operatorname{supp}(\mathbf{x})=\{\pi(i), \pi(j)\}$ для любого $\mathbf{x} \in \mathscr{Q}^{-1}(\mathbf{y})$, т.е.

$$
\mathscr{Q}^{-1}\left(\operatorname{int}\left(\Gamma_{\mathbf{e}_{i} \mathbf{e}_{j}}\right)\right)=\operatorname{int}\left(\Gamma_{\mathbf{e}_{\pi(i)} \mathbf{e}_{\pi(j)}}\right) \quad \text { для всех } i, j \in \mathbf{I}_{m} .
$$

Теорема доказана.

\footnotetext{
${ }^{1}$ Приведенное выше короткое доказательство было предложено анонимным рецензентом. В первоначальном доказательстве использовались степени отображений. Нетрудно показать, что заданное отображение гомотопно тождественному отображению и имеет степень 1.
} 
3. Операторы Лотки-Вольтерра. Квадратичный стохастический оператор $\mathscr{V}$ : $\mathbb{S}^{m-1} \rightarrow \mathbb{S}^{m-1}$ называется оператором Лотки-Вольтерра, если

$$
\operatorname{null}\left(\mathbf{p}_{i j \bullet}\right) \supset \mathbf{I}_{m} \backslash\{i, j\} \quad \text { для любых } i, j \in \mathbf{I}_{m}
$$

(см. [1], [8]). Для квадратичного стохастического оператора Лотки-Вольтерра $\mathscr{V}: \mathbb{S}^{m-1} \rightarrow$ $\mathbb{S}^{m-1}$ имеем $\mathbf{p}_{i i} \bullet=\mathbf{e}_{i}$ и $\operatorname{supp}\left(\mathbf{p}_{i j \bullet}\right) \subset\{i, j\}$ при всех $i \neq j$. Таким образом, в этом случае квадратичный стохастический оператор Лотки-Вольтерра имеет вид

$$
\mathscr{V}(\mathbf{x})=\sum_{i=1}^{m} x_{i}^{2} \mathbf{e}_{i}+2 \sum_{i \neq j} x_{i} x_{j} \mathbf{p}_{i j} \bullet
$$

Нетрудно проверить, что квадратичный стохастический оператор является оператором Лотки-Вольтерра тогда и только тогда, когда любое не более чем двухэлементное множество $\alpha \subset \mathbf{I}_{m}$ является поглощающим.

Таким образом, справедливо следующее утверждение.

Теорема 2. Квадратичный стохастический оператор $\mathscr{Q}: \mathbb{S}^{m-1} \rightarrow \mathbb{S}^{m-1}$ сюрвективен тогда и только тогда, когда существуют перестановочная матрица П и квадратичный стохастический оператор Лотки-Вольтерра $\mathscr{V}: \mathbb{S}^{m-1} \rightarrow \mathbb{S}^{m-1}$, для которых $\mathscr{Q}(\mathbf{x})=$ $\Pi \mathscr{V}(\mathbf{x})$ при любом $\mathbf{x} \in \mathbb{S}^{m-1}$.

В статье [1] содержится следующее описание всех квадратичных стохастических автогомеоморфизмов симплекса.

Tеорема 3 [1]. Квадратичный стохастический оператор $\mathscr{Q}: \mathbb{S}^{m-1} \rightarrow \mathbb{S}^{m-1}$ является автоморфизмом симплекса тогда и только тогда, когда существуют перестановочная матрица П и квадратичный стохастический оператор Лотки-Вольтерра $\mathscr{V}: \mathbb{S}^{m-1} \rightarrow$ $\mathbb{S}^{m-1}$, для которьх $\mathscr{Q}(\mathbf{x})=\Pi \mathscr{V}(\mathbf{x})$ при любом $\mathbf{x} \in \mathbb{S}^{m-1}$.

Отсюда вытекает следующее утверждение.

СлЕдСтвиЕ. Квадратичный стохастический оператор на симплексе, связанный с кубической стохастической матрицей, сюрвективен тогда и толъко тогда, когда он биективен (является автоморфизмом симплекса).

Следующая теорема представляет независимый интерес; ее можно доказать с помощью техники из доказательства леммы 1.

Напомним (см. [9], [10]), что отображение $\mathscr{V}_{\mathbf{F}}: \mathbb{S}^{m-1} \rightarrow \mathbb{S}^{m-1}$, определенное правилом $\mathscr{V}_{\mathbf{F}}(\mathbf{x})=\mathbf{x}^{\prime}$, где

$$
x_{k}^{\prime}=x_{k}\left(1+f_{k}(\mathbf{x})\right), \quad k=1, \ldots, m,
$$

называется оператором Лотки-Вольтерра, если отображение $\mathbf{F} \equiv\left(f_{1}, f_{2}, \ldots, f_{m}\right): \mathbb{S}^{m-1} \rightarrow$ $\mathbb{R}^{m}$ удовлетворяет следующим условиям:

1) отображение $\mathbf{F}: \mathbb{S}^{m-1} \rightarrow \mathbb{R}^{m}$ непрерывно;

2) $f_{k}(\mathbf{x}) \geqslant-1$ для всех $\mathbf{x} \in \mathscr{S}^{m-1}$ и $k=1, \ldots, m$;

3) $\sum_{k=1}^{m} x_{k} f_{k}(\mathbf{x})=0$ для всех $\mathbf{x} \in \mathbb{S}^{m-1}$;

4) для любого $\alpha \subset \mathbf{I}_{m}$ выполнено неравенство $f_{k}(\mathbf{x})>-1$ при всех $x \in \operatorname{int} \Gamma_{\alpha}, k \in \alpha$.

Теорема 4. Оператор Лотки-Вольтерра $\mathscr{V} / \mathbf{F}$ на симплексе сюргективен.

Следует отметить, что оператор Лотки-Вольтерра определяет странную (неэргодическую) динамику (см. [11]-[13]).

Автор выражает благодарность Международному центру теоретической физики (ICTP) г. Триесты (Италия), где была написана эта статья в рамках плана сотрудничества с молодыми учеными, за приглашение и гостеприимство. 


\section{СПИСОК ЦИТИРОВАННОЙ ЛИТЕРАТУРЫ}

[1] R. Ganikhodzhaev, F. Mukhamedov, U. Rozikov, Infin. Dimens. Anal. Quantum Probab. Relat. Top., 14:2 (2011), 279-335. [2] А.В. Арутюнов, Функи. анализ и его прил., 46:3 (2012), 81-84. [3] Ю.И. Любич, Математические структуры в популяиионной генетике, Наукова думка, Киев, 1983. [4] N. Ganikhodjaev, M. Saburov, U. Jamilov, Appl. Math. Inf. Sci., 7:5 (2013), 1721-1729. [5] N. Ganikhodjaev, M. Saburov, A. M. Nawi, The Scientific World Journal, 2014 (2014), Article ID 835069. [6] M. Saburov, Kh. Saburov, Science Asia, 40:4 (2014), 306-312. [7] M. Saburov, Kh. Saburov, Int. J. Control, Automat. Systems, 12:6 (2014), 1276-1282. [8] F. Mukhamedov, M. Saburov, Appl. Math. Inf. Sci., 4:1 (2010), 47-62. [9] Р. Н. Ганиходжаев, М. Х. Сабуров, ЖКурн. СФУ. Сер. Матем. и физ., 1:2 (2008), 188-196. [10] F. Mukhamedov, M. Saburov, Bull. Malays. Math. Sci. Soc. (2), 37:1 (2014), 59-64. [11] M. Saburov, World Appl. Sci. J., 21:1 (2013), 94-97. [12] M. Saburov, Math. Notes, 97:5 (2015), 759-763. [13] M. Saburov, Ann. Funct. Anal., 6:4 (2015), 247-254.

М. Сабуров

International Islamic University Malaysia

E-mail: msaburov@gmail.com
Поступило

08.07.2015

Исправленный вариант

07.10 .2015 